\title{
Multiwavelength Gamma-Ray Bursts Observations with ECLAIRs
}

\author{
Diego Götz on behalf of the ECLAIRs collaboration (CEA Saclay, CESR \\ Toulouse, APC Paris, MIT Boston) \\ CEA - SAp/DAPNIA/DSM, Orme des Merisiers, F-91191, Gif sur Yvette, France \\ e-mail:diego.gotz@cea.fr
}

\begin{abstract}
ECLAIRs is the next space borne instrument that will be fully dedicated to multiwavelength studies of Gamma-Ray Bursts (GRBs). It consists of a coded mask telescope with a wide $\left(\sim 2 \mathrm{sr}\right.$ ) field of view, made of 6400 CdTe pixels $\left(\sim 1000 \mathrm{~cm}^{2}\right)$, which will work in the 4$300 \mathrm{keV}$ energy band. It is expected to localise $\sim 80 \mathrm{GRBs} / \mathrm{yr}$, thanks to the on-board real time event processing. The GRBs (and other transients) coordinates will be distributed within a few seconds from the onset of the burst with a typical uncertainty of $\sim 5-10$ arcmin. The detection system will also include a soft X-ray camera (1-10 keV) allowing to study in detail the prompt soft X-ray emission and to reduce the error box for about half of the GRBs seen by ECLAIRs to $\sim 30$ arcsec. ECLAIRs is expected to be flown in late 2011 and to be the only instrument capable of providing GRB triggers with sufficient localisation accuracy for GRB follow-up observations with the powerful ground based spectroscopic telescopes available by then. We will present the current status of the ECLAIRs project and its possible developments.
\end{abstract}

Keywords: Instrumentation: detectors ; Gamma rays: bursts

PACS: $98.70 . \mathrm{Rz} ; 95.55 . \mathrm{Ka}$

\section{INTRODUCTION}

Ever since their discovery [Klebesadel et al. 1973], Gamma-Ray Bursts (GRBs) have been a puzzling mystery, mostly because of their short durations and the apparent lack of counterparts at other wavelengths. A breakthrough in this field came thanks to the Italian-Dutch satellite BeppoSAX, which had the capability to localise the bursts' prompt emission with a precision of a few arcminutes within a few hours. This led to the discovery of the afterglow emission at lower energies, initially in X-rays [Costa et al. 1997] and subsequently at optical [van Paradijs et al. 1997] and radio [Frail et al. 1997] wavelengths, which allowed the redshift of these objects to be measured, and firmly established the cosmological nature of GRBs.

Due to the limited duration and the fading character of the afterglow emission, the prompt distribution of GRBs coordinates to the scientific community is a high priority. After the end of the BeppoSAX mission this task has been accomplished mainly by HETE-II [Ricker et al. 2002], and INTEGRAL through to the INTEGRAL Burst Alert System (IBAS; [Mereghetti et al. 2003]). Since 2005, thanks to the Swift satellite [Barthelmy et al. 2005], and to its capability to pinpoint the postions of GRBs to a few arcseconds in few minutes, the GRB field is progressing quickly. In particular the association between long GRBs and the death of massive $\left(\gtrsim 20 \mathrm{M}_{\odot}\right)$ stars is becoming stronger, and the study of the early X-ray afterglows has shown new flaring phases, 
which were unknown before Swift. In addition, thanks to its good sensitivity, Swift is localising a growing number of GRBs at high redshift, the most distant one being 050904 at a redshift of 6.29 [Watson et al. 2006].

Although considerable progress in the understanding of GRBs has been made in the last years, some crucial points still need some further investigation: the nature of the prompt emission, its relationship to the afterglow, the nature of the central engine, the nature of the progenitor star, in particular in connection to cosmological studies. In order to answer to these questions, it is fundmental to collect as much information as possible on every single GRB. The ECLAIRs project is being developed for this scope.

\section{THE ECLAIRS PROJECT}

The goal of the ECLAIRs project is conceived to detect during its lifetime at least 200 GRBs, of all types, short and long, and in particular the ones with a soft spectrum, potentially the most distant ones [Schanne et al. 2005, Schanne et al. 2006]. The GRBs should be observed also before and after the main event, in particular to study the spectrum of a possible precursor. These GRBs should be observed on a broad energy range in order to study the prompt X-ray component, and to constrain as much as possible the bursts "peak energy", $E_{p}$, namely the energy at which a GRB emits the bulk of its energy. The determination of $E_{p}$ is very important since in the last years some correlations have been found between this quantity and the isotropic equivalent energy, $E_{i s o}$ [Amati et al. 2002], and between $E_{p}$ and $E_{i s o}(1-\cos \theta)$, the collimation corrected energy [Ghirlanda et al. 2004]. The scatter around the latter correlation is tight, and suggests to use the GRBs as cosmological tools. Indeed Ghirlanda \& Ghisellini [2004] estimate that a sample of 12 well studied GRBs with redshifts between 0.9 and 1.1 are enough to calibrate their correlation, and its application to 150 bursts gives good constraints on the cosmological parameters $\Omega_{M}$ and $\Omega_{\Lambda}$. These constraints are complementary in the $\left(\Omega_{M}, \Omega_{\Lambda}\right)$ plane to the ones obtained from the cosmic microwave background and from the Supernovae of type I.

Another fundamental aspect of the project is to ensure the possibility of a follow-up from large ground based optical telescopes for at least $75 \%$ of the detected bursts. The observation plan will in fact be adjusted in order to have at least one 8-m-class telecope that covers a large fraction of ECLAIRs field of view. This is crucial in order to be able to determine the redshift of GRBs, and hence their luminosity, in particular, in view of the second generation spectrometers that will be available in 2011, like X-Shooter. In order to allow for the follow up, ECLAIRs will have to provide a coarse (10 arcmin) localisation within $10 \mathrm{~s}$ from the burst onset. This information can be exploited from ground based robotic telescopes. For half of the GRBs a position with an accuracy of 30 arcsec should be provided within the same time interval. Within 1 minute the best available information should reach all the major telescopes on Earth. 


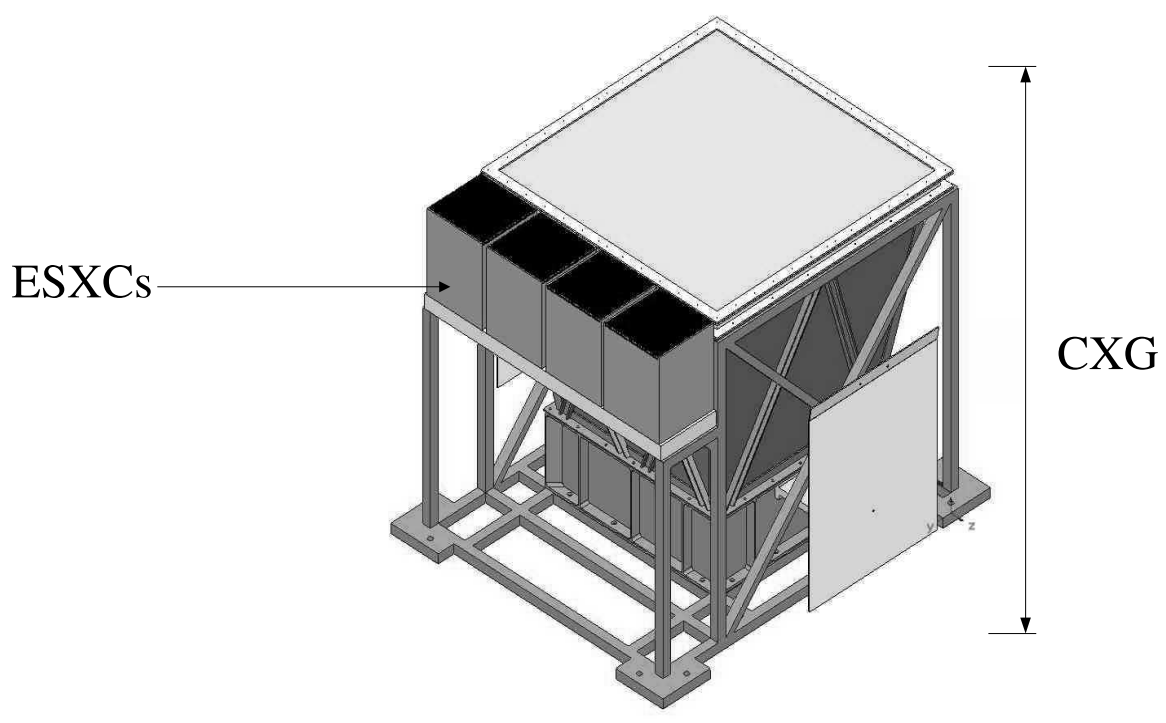

FIGURE 1. The ECLAIRs telescope

\section{The Instruments}

The ECLAIRs telescope will be composed by the $\mathrm{X}$ and Gamma-Ray Camera (CXG, the trigger device) and a Soft X-ray Camera (ESXC), see Fig. 1. Besides the space borne instruments the mission includes also two dedicated Ground Follow-up robotic Telescopes (GFT) to study the prompt and early afterglow emission of GRBs.

The CXG and ESXC will have a wide field of view of $\sim 2$ sr. They are both coded mask telescopes and will be operating in th 4-300 keV and 1-10 keV energy range respectively. The CXG will be made of $80 \times 80$ pixels of CdTe yielding an effective area of $1024 \mathrm{~cm}^{2}$, and will be able to localise all the bursts in its field of view within 10 arcmin (for a $5 \sigma$ source). The ESXC camera, triggered by the CXG with its coarse position, will be able to localise the prompt GRBs emission, for about half of the burst, within $30 \operatorname{arcsec}(5 \sigma)$. This instrument will be based on the experience of the soft $\mathrm{X}$-ray camera on board HETE-II. The characteristics of the ECLAIRs instruments are summarised in Table 1 .

In the framework of the ECLAIRs project the addition of more instruments is being studied in order to extend the energy band over which the GRBs can be studied: as stated earlier, the measurement of $E_{p}$ is of primary importance. Hence a gamma-ray detector, extending the energy range up to a few $\mathrm{MeV}$, is currently being studied. Besides the GFT 
TABLE 1. Characteristics of ECLAIRs instruments

\begin{tabular}{|c|c|c|}
\hline & ECLAIRs/CXG & ECLAIRs/ESXC \\
\hline Energy range & $4-300 \mathrm{keV}$ & $1-10 \mathrm{keV}$ \\
\hline Field of view & $2 \mathrm{sr}$ & $2 \mathrm{sr}$ \\
\hline Sensitive area & $1024 \mathrm{~cm}^{2} \mathrm{CdTe}$ & $96 \mathrm{~cm}^{2} \mathrm{Si}$ \\
\hline Mask open fraction & $30 \%$ & $20 \%$ \\
\hline Temporal resolution & $10 \mu \mathrm{s}$ & $100 \mathrm{~ms}$ \\
\hline Source localisation & 10 arcmin for $5 \sigma$ & 30 arcsec for $5 \sigma$ \\
\hline
\end{tabular}

a wide field optical camera could be placed on board in order to study the prompt optical emission.

\section{Number of GRBs per year}

With the current mission design one expects that ECLAIRs will localise about 80 GRBs per year. This calculation is based on the instrument geometry, and on a background evaluation made under the hypothesis that between 4 and $50 \mathrm{keV}$ (the CXG triggering band) the background is dominated, in absence of strong sources, by the cosmic diffuse X-ray background. An analytic expression of it can be found in [Gruber et al. 1999], and is

$$
7.877 \times E^{-0.29} e^{-E / 41.13} \frac{\mathrm{keV}}{\mathrm{keV} \mathrm{cm}^{2} \mathrm{~s} \mathrm{sr}}
$$

By numerically integrating Eq. 1 between 4 and $50 \mathrm{keV}$, multiplying for the detector geometrical area $\left(\mathrm{A}_{e f f}=0.4 \times 0.4 \times 80 \times 80 \mathrm{~cm}^{2}=1024 \mathrm{~cm}^{2}\right)$, and taking the aperture and the average efficiency into account (the CdTe efficiency is practically $100 \%$ in this energy range), one obtains a background value of 2050 counts $\mathrm{s}^{-1} \mathrm{sr}^{-1}$. To evaluate the number of GRBs in the sky we numerically integrated the peak flux density distribution derived over $1 \mathrm{~s}$ with BATSE [Paciesas et al. 1999], $\mathrm{N}(\mathrm{P})$, where $\mathrm{N}$ is the number of bursts detected per year and above a given peak flux $\mathrm{P}$. We then took into account the CXG livetime which is mainly limited by the passages above the southern Atlantic anomaly and by the time the earth enters the field of view (see below). In the hypothesis of a pure antisolar pointing and an equatorial orbit with an inclination of $30^{\circ}$ one obtains, on average, that for $64 \%$ of the orbit we have a field of view not occulted by the earth. We will call this factor, $l t=0.59$. The triggering energy band of the $C X G$ is different from the BATSE one (50-300 keV). So, first of all one has to convert the BATSE fluxes into CXG fluxes and this has been done by assuming a GRB spectrum, described by a Band function [Band et al. 1993], with average parameters $\left(E_{p}=200 \mathrm{keV}, \beta=-2.25, \alpha=-\right.$ 1) [Preece 2000]. This introduces a multiplicative factor of $e c=2.45$ on the Log N-Log P. In addition recent missions like BeppoSAX, HETE-II, INTEGRAL and Swift, have shown that there is an entire class of GRBs, the so called, X-Ray Flashes (XRFs), that were too soft to be detected by BATSE. So we have to add another multiplicative factor that takes 
this into account, and its value is $n_{X} \sim 1.55\left(=\left(\mathrm{N}_{G R B}+\mathrm{N}_{X R R}+\mathrm{N}_{X R F}\right) /\left(\mathrm{N}_{G R B}+\mathrm{N}_{X R R}\right)\right)$, as derived by HETE-II data [Sakamoto et al. 2005].

The CXG sensitivity limit in the $4-50 \mathrm{keV}$ band for a $5 \sigma$ detection in the totally coded field of view is $S_{\lim 0} \sim 0.7 \mathrm{ph} \mathrm{cm}^{-2} \mathrm{~s}^{-1}$, based on the background value derived above and taking into account that a square $\mathrm{cm}$ of detector sees a different sky fraction depending on its position on the detector (a variation between $\sim 0.9$ and $\sim 1 \mathrm{sr}$ ).

By taking the above factors, the variation of the effective area as a function of the offaxis angles of the instrument $\mathrm{A}_{e f f}\left(\theta_{X}, \theta_{Y}\right)$, the relative sensitivity variation, $\mathrm{S}_{\text {lim }}\left(\theta_{X}, \theta_{Y}\right.$, and the variation of the solid angle seen by the detector in each direction of the field of view, $\Omega\left(\theta_{X}, \theta_{Y}\right)$, into account, one derives the number of GRBs per year observable with the $\mathrm{CXG}$ in the following way:

$$
N_{G R B}=\operatorname{lt} n_{X} \int_{\theta_{X}} \int_{\theta_{Y}} S_{l i m}\left(\theta_{X}, \theta_{Y}\right) \cdot \Omega\left(\theta_{X}, \theta_{Y}\right) / 4 \pi \int_{S_{l i m}\left(\theta_{X}, \theta_{Y}\right)}^{\infty} N(P \cdot e c) d P d \theta_{X} d \theta_{Y}
$$

with $\theta_{X}$ and $\theta_{Y}$ being the off-axis angles that describe the field of view. The result is 76 bursts/year.

\section{The Alert Strategy}

The positions of the transients detected by the ECLAIRs telescope will be derived on board. A dedicated real-time processing unit, UTS, will be present. The UTS will look for significant excesses in the CXG light curve simultaneously on different time scales, energy bands and detector portions. In case of a positive excess the imaging process will be invoked and, if a previously uncatalogued source is found, an alert will be generated. This alert will be transmitted promptly to the ground, so that robotic telescopes can quickly react. At the same time the infomation will be passed to the ESXC in order to refine the position, if possible. At the same time images on different energy bands and timescales are produced continuously and compared to previously recorded background images in order to spot new transients that could have been missed by the previous algorithms. The latter, introduced for the first time in the IBAS system, is particularly effective in detecting long and "slowly rising" bursts, like 050904. The positions derived on board with ECLAIRs will be distributed to the ground via a VHF network, which will be an extension of the one used today by HETE-II.

\section{The Follow-up}

The pointing strategy and the orbit of ECLAIRs will be adapted in order to have a large chance of follow-up by the largest ground based telescopes. The launcher should place ECLAIRs into a circular orbit at $600 \mathrm{~km}$ altitude with an inclination of $30^{\circ}$. During most of the orbit the satellite will perform an anti-solar pointing. In addition the avoidance of regions of bright X-ray sources, in particular Sco X-1, can be accommodated by varying the angle between the orbital plane and the pointing direction. 


\section{CONCLUSIONS}

The ECLAIRs system could be incorporated in a next mission dedicated to GRBs. Besides GRBs, this mission will include the study of all the transient phenomena in the high-energy sky, such as X-ray bursts, Soft Gamma-Ray Repeaters, AGNs, Supernovae, Novae, etc.

Although thanks to Swift considerable progress has been made in GRB science, some aspects will be explored further thanks to ECLAIRs. These aspects include

- the study of the prompt X-ray emission associated to GRBs

- the detection of a large sample of soft GRBs, potentially the most distant ones, thanks to the 4-50 keV triggering band

- the broad band spectra (from $1 \mathrm{keV}$ to a few MeV)

- the prompt (and/or precursive) optical emission

In addition the ECLAIRs pointing strategy will be crucial to ensure the most favourable conditions for follow-up studies from ground based telescopes.

\section{ACKNOWLEDGMENTS}

DG acknowledges support from the French Space Agency (CNES).

\section{REFERENCES}

Amati et al. 2002. Amati, L., et al. 2002, A\&A, 390, 81

Band et al. 1993. Band D., et al. 1993, ApJ, 413, 281

Barthelmy et al. 2005. Barthelmy, S. D., et al. 2005, Space Science Reviews, 120, 143

Costa et al. 1997. Costa, E., Frontera, F., Heise, J., et al. 1997, Nature, 387, 783

Frail et al. 1997. Frail, D. et al. 1997, Nature, 389, 261

Ghirlanda et al. 2004. Ghirlanda, G., Ghisellini, G., \& Lazzati, D. 2004, ApJ, 616, 331

Ghirlanda \& Ghisellini 2004. Ghirlanda, G., \& Ghisellini, G. 2004, in proceedings of the workshop "Science with the New Generation of High-Energy Gamma-Ray Experiments", Cividale del Friuli (Italy), astro-ph/0602498

Gruber et al. 1999. Gruber D.E., et al. 1999, ApJ, 520, 124

Klebesadel et al. 1973. Klebesadel, R. et al. 1973, ApJ, 182, L85

Mereghetti et al. 2003. Mereghetti, S., Götz, D., Borkowski, J., et al. 2003, A\&A, 411, L291

Paciesas et al. 1999. Paciesas W.S., et al. 1999 ApJS, 122, 465

Preece 2000. Preece R.D. et al., 2000, ApJS, 126, 19

Ricker et al. 2002. Ricker, G., Hurley, K., Lamb, D., et al. 2002, ApJ, 571, L127

Sakamoto et al. 2005. Sakamoto T. et al., 2005, ApJS, 629, 311

Schanne et al. 2005. Schanne, S., Atteia, J.-L., Barret, D., et al. 2005, IEEE Transact. Nucl. Sci, 52 , 2778

Schanne et al. 2006. Schanne, S., Atteia, J.-L., Barret, D., et al. 2006, NIM-A, in press

van Paradijs et al. 1997. van Paradijs, J., Groot, P.J., Galama, T., et al. 1997, Nature, 386, 686

Watson et al. 2006. Watson, D., Reeves, J.N., Hjorth, J, et al. 2006, ApJ, 637, L69 